\title{
À propos de l'ouvrage d'Alain Pavé Comprendre la biodiversité. Vrais problèmes et idées fausses
}

\author{
Jacques Blondel* \\ Écologie, ancien président du conseil scientifique de l'Institut français de la biodiversité, CNRS, UMR CEFE, Montpellier, \\ France
}

Depuis 50 ans, la somme des recherches menées en écologie, au sein d'institutions et de programmes internationaux, est impressionnante, en France comme ailleurs. Alain Pavé (Université de Lyon), auteur de l'ouvrage Comprendre la biodiversité. Vrais problèmes et idées fausses, dont il est question ici, et Jacques Blondel (CNRS), qui nous propose son regard sur ce livre, font partie de ces chercheurs, à la fois rigoureux et engagés, dont les travaux et le rôle institutionnel ont eu une réelle portée dans le champ de l'écologie.

Ils ont tous les deux apporté leur contribution, à partir de leurs domaines de compétence, à la construction de cette science multiple aux nombreuses interactions avec des disciplines voisines, mais aussi avec l'ingénierie, la modélisation, les statistiques et les sciences politiques. En s'appuyant sur cette base solide, les écologues peuvent aujourd'hui affronter l'hypothèse catastrophiste d'une $6^{\mathrm{e}}$ extinction, s'interroger sur l'entrée des questions de biodiversité dans la sphère marchande et participer à l'émergence d'une coordination et d'une politique mondiales de la biodiversité à travers l'IPBES.

Le texte de Jacques Blondel à propos de l'ouvrage d'Alain Pavé nous apparaît comme un bon point de départ pour entamer un débat sur la question plurielle de la biodiversité : quel meilleur exemple pour réfléchir sur l'interdisciplinarité des années 2020 ? La vivacité de certaines analyses présentes ici ne doit pas occulter la profondeur des points de vue, qui, nous l'espérons, inciteront à nous proposer de nouveaux textes sur ce débat très actuel.

La Rédaction

\begin{abstract}
Résumé - L'objet du livre d'Alain Pavé, Comprendre la biodiversité. Vrais problèmes et idées fausses, paru en 2019 au Seuil, est de retracer l'histoire du concept de biodiversité qui exprime et concrétise l'inquiétude que suscite son déclin. Son propos est d'examiner comment ce terme polysémique est utilisé, parfois à tort et à travers, étant précisé que le concept intéresse à la fois les sciences de la nature et les sciences de l'homme et de la société dans un souci d'étude, de valorisation et de partage des avantages procurés aux sociétés par la biodiversité.

Construit en huit chapitres, l'ouvrage part d'exemples et d'expériences, développe la question des inventaires de biodiversité et des méthodes et métriques utilisées pour la qualifier et la quantifier. L'évolution de la biodiversité au cours des 600 derniers millions d'années est abordée à partir de la phylogénétique moléculaire et le rôle du hasard dans les variations de biodiversité globale est souligné. Quant à la projection dans le futur, elle est abordée par une réflexion critique sur l'existence même d'un déclin de la biodiversité. La manière dont les écologues parlent de la biodiversité, l'étudient et prétendent la conserver est l'objet d'une sévère remise en cause de la démarche scientifique actuelle. D'où la nécessité d'une reconstruction de l'écologie scientifique, y compris dans le domaine de la conservation jugée pratiquée d'une façon passéiste, voire fixiste. Puis l'auteur revient sur le thème de la modélisation qui permet d'intégrer le hasard dans la compréhension de l'évolution des systèmes biologiques.

L'ouvrage propose une lecture très personnelle du concept de biodiversité et dénonce le catastrophisme ambiant qui se nourrit d'affirmations jugées péremptoires et trop pessimistes, y compris quand on parle d'une $6^{\mathrm{e}}$ crise d'extinction de masse dont la réalité est contestée. Mais il est loin de faire justice d'une immense littérature qui traduit le dynamisme de la recherche en écologie et évolution. Cette très faible pénétration dans la littérature entraîne souvent le discours dans des considérations naïves et dépassées sur des concepts fondateurs de l'écologie. L'ouvrage paraît être victime du syndrome, bien connu des
\end{abstract}

\footnotetext{
* Auteur correspondant : jac.blondel@wanadoo.fr
} 
philosophes des sciences, de la fascination du chercheur pour son outil de travail et la culture de l'effet que ses écrits produisent dans le «grand cirque de la science». Partielle et partiale, la bibliographie ne fait pratiquement jamais appel aux milliers d'articles publiés chaque année par des milliers d'auteurs dans les grandes revues internationales, y compris celles qui font périodiquement le point sur l'état des connaissances sur ces questions.

Mots-clés : biodiversité / hasard / évolution / modélisation / dynamique des populations / phylogénétique moléculaire / extinction

\begin{abstract}
About Alain Pavé's book Comprendre la biodiversité. Vrais problèmes et idées fausses. This book aims at understanding the biodiversity concept which encompasses the many concerns raised by its decline. One question is to review the various ways whereby this polysemous word is rightly or wrongly used by both life sciences and sciences of humans and societies. The problem is to study biodiversity for the sake of development as well as sharing of the many benefits biodiversity provides to societies.

Starting from examples and experience the book includes eight chapters and develops the question of inventories and the methods and metrics used for qualify and quantify biodiversity. Evolution of life in the course of the 600 past million years is mostly addressed from molecular phylogenetics, with a focus on hazard as a driver of variation in biodiversity levels over time. About the future of biodiversity, the author critically questions the reality of a sixth global extinction crisis. The current approaches to biodiversity study and conservation are critically challenged so that the author calls for a complete refoundation of this discipline. This is especially true because scientists and conservationists are said to work in an old fashion, being eventually fixists. Then the author comes back to his own field of research, namely modelling, which allows including hazard to interpret and understand the dynamics and evolution of living systems.

A very personal reading of the biodiversity concept is offered in this book which strongly declaims against the currently widespread catastrophism as well as peremptory and pessimistic claims, including about the alleged sixth extinction crisis which is far from proven. However the book is far from making justice of the huge literature which expresses the dynamism of research in ecology, evolution and conservation. As a result, the weak support of the relevant literature often makes reasoning and demonstrations out of date and naive, quite far from the current standards of ecological theory, including regarding founding concepts of ecology. The book seems to suffer from a syndrome which is well known by philosophers of science, namely the fascination of researchers in their working tool and the search for the effects of their writings in the "big circus of science". The literature is biased and quite partial, and almost never makes use and justice of the thousands papers published annually by thousands authors in well-known international journals, including those that periodically make the state of the art in themes discussed in the book.
\end{abstract}

Keywords: biodiversity / hazard / evolution / modelling / population dynamics / molecular phylogenetics / extinction

En ces temps de désordres climatiques et d'effondrement de la diversité du vivant, un ouvrage scientifique sur la biodiversité est toujours très attendu et prend une résonance particulière, tant son impact sur nos contemporains peut être fort, surtout quand il s'adresse au grand public. À condition bien sûr qu'il réponde à certaines exigences, en particulier celle du respect de la légitimité d'une pluralité de regards sur ce concept polysémique de biodiversité, objet de centaines de livres. Dans l'ouvrage Comprendre la biodiversité. Vrais problèmes et idées fausses, paru en 2019 au Seuil, les regards d'Alain Pavé, son auteur, sont ceux d'un spécialiste de la modélisation des systèmes vivants ayant à son actif de nombreux livres et travaux de biostatistiques, de mathématiques et de modélisation, appliqués à la structure et à la dynamique des communautés et des populations.

\section{Les regards de l'auteur sur la biodiversité}

Partant de LUCA (Last Universal Common Ancestor), pour aboutir à ce « grand bazar » qu'est la diversité actuelle du vivant, Alain Pavé propose de retracer la saga du concept de biodiversité «ballotté entre des visions scientifiques à l'image de celle ayant permis la reconstruction du génome de LUCA, et des récits plus ou moins fantasmés, comme ceux des dessins animés. » Soulignant que le néologisme «biodiversité», forgé dans les années 1980, exprime et concrétise l'inquiétude que suscitent l'épuisement des ressources vivantes et la dégradation de leur environnement, l'auteur a construit son ouvrage en huit chapitres. Après un bref «tour d'horizon» qui annonce le ton que prendra le livre, Alain Pavé part «d'exemples et d'expériences» puis chemine à travers «l'histoire quelque peu brinquebalante » du concept, mais sans en proposer une lecture épistémologique. Laquelle permettrait de faire une utile distinction entre l'expression « diversité de la vie » qui rend compte d'un état, celui de la diversité du monde vivant, et le mot «biodiversité » qui considère cette diversité en tant qu'elle est instrumentalisée par les humains pour le meilleur, mais aussi pour le pire (Blondel, 2012). Il est opportunément rappelé que ce que recouvre ce néologisme, dont la polysémie est 
soulignée, intéresse à la fois les sciences de la nature et les sciences de l'homme et de la société. Le chapitre suivant, «On compte beaucoup plus ou moins bien, et qu'en faiton?», développe la question de la quantification des entités de biodiversité, des gènes aux écosystèmes et des méthodes et métriques utilisées pour les qualifier et les quantifier. Le quatrième chapitre « Et si on parlait un peu d'évolution" introduit le lecteur dans l'histoire de la biodiversité sur le temps long de l'évolution, insistant sur le rôle du hasard mais sans citer Ernst Mayr ni Jacques Monod, ce qui est étonnant, surtout de la part d'un auteur français qui, de surcroît, a écrit, paraphrasant Le hasard et la nécessité de Jacques Monod, un ouvrage intitulé $L a$ nécessité $d u$ hasard! D'intéressants développements mathématiques sont proposés sur l'histoire et les modalités des variations de biodiversité globale au cours des 600 derniers millions d'années. Cette remontée dans le temps illustre la profondeur temporelle de cette histoire mais rien n'est dit sur «l'origination» des taxons actuels qui, à la lecture des phylogénies moléculaires dont on dispose aujourd'hui pour la plupart des grands groupes végétaux et animaux, s'enracine pour la majorité d'entre eux dans un temps beaucoup plus profond que ce qu'on a longtemps pensé. Le cinquième chapitre «Prévisions de la biodiversité : vers une sixième grande extinction?» discute l'opportunité de parler de crise d'extinction s'agissant des variations actuellement observées de la biodiversité. Les deux chapitres suivants «La biodiversité comment en parle-t-on» et "Déconstruire et reconstruire l'écologie scientifique?» examinent de façon très critique le discours actuel sur l'état de la biodiversité et s'interrogent sur l'opportunité de la conserver. Puis, les rapports entre humains et biodiversité sont examinés sur les plans sociologique, juridique et économique. Conséquence logique de l'esprit général du livre qui est une remise en cause radicale de la démarche scientifique telle qu'elle est pratiquée par la communauté scientifique, Alain Pavé se dit convaincu de la nécessité d'une refondation de l'écologie scientifique. Le dernier chapitre «Ce que dit la biodiversité sur le hasard et le hasard sur la biodiversité » revient sur ce thème de prédilection de l'auteur qu'est la modélisation qui a permis d'intégrer le hasard dans la compréhension de la dynamique et de l'évolution des systèmes biologiques. Une intéressante rétrospective est offerte sur la façon dont le hasard, mais aussi la contingence, sont perçus dans l'histoire des idées, de Pascal à Poincaré, en passant, bien sûr, par Spinoza. On aurait pu trouver ici, outre une référence à la pensée de Jacques Monod, un rappel des travaux pénétrants de Jean Gayon qui évoque les trois sens de la notion de hasard intervenant dans la théorie de l'évolution: la notion ordinaire de chance, la notion probabiliste d'aléatoire et la notion épistémologique de contingence relativement à un système théorique.

\section{Polysémie des sens, pluralité des lectures}

Sur le fond, l'ouvrage propose une lecture très personnelle du concept de biodiversité et se démarque radicalement du discours actuel de la très grande majorité des chercheurs impliqués dans ces thématiques. Trois commentaires seulement: le premier porte sur l'esprit général du livre qui fustige un catastrophisme ambiant se nourrissant d'affirmations jugées péremptoires et trop pessimistes, y compris quand les écologistes parlent d'une $6^{\mathrm{e}}$ crise d'extinction de masse. L'argumentaire mathématique, sorte de deus ex machina surplombant l'ensemble de l'ouvrage, est utilisé pour dénoncer le catastrophisme des prophètes de malheur qui proclament urbi et orbi l'effondrement de la biodiversité, alors que, selon l'auteur, ce déclin n'est qu'apparent car dû à des biais statistiques (par exemple, p. 115), d'autant plus que «[1]es estimations de la biodiversité sont souvent acrobatiques, parfois fantaisistes» (p. 119).

Mon deuxième commentaire porte sur le sens donné par Alain Pavé au mot biodiversité. Sans développer la nature polysémique du terme qui n'est défini formellement qu'une fois et de façon très personnelle et expéditive comme «ensembles d'êtres vivants de même catégorie », l'auteur souligne avec raison que la science ne l'épuise pas puisque la biodiversité est aussi politique, économique, philosophique, culturelle, voire religieuse, mais il n'aborde qu'une partie seulement des composantes strictement biologiques du concept, ignorant ces autres dimensions d'un grand intérêt théorique et pratique que sont la «biodiversité taxinomique», la «biodiversité phylogénétique», la «biodiversitédifférenciation», la «biodiversité fonctionnelle», la «biodiversité-processus», mais aussi la «biodiversitéservices ». Une manière de voir la biodiversité actuelle, surtout quand on développe sa composante évolutive comme le fait l'auteur, est de la considérer comme un enchaînement de processus plutôt que comme une suite d'états. Car la biodiversité est un processus constant d'apparition et de disparition de formes de vie, à toutes les échelles de diversité organique, des individus aux populations, aux espèces puis aux assemblages d'espèces, lesquels sont tout sauf un "grand bazar»!

S'agissant de la «biodiversité-services », on aurait pu s'attendre à voir une analyse un peu approfondie de ce nouveau Graal de la biodiversité que sont les notions de capital naturel, décliné en services écosystémiques (services d'approvisionnement, de régulation, de support, culturels), mais aussi de «disservices». Cette nouvelle rhétorique sur l'économie de la nature, largement mise sur le devant de la scène par l'Évaluation des écosystèmes pour le millénaire (MEA [Millennium Ecosystem Assessment], 2005), réalisée dans le sillage 
de la conférence de Rio de Janeiro de 1992 ou « Sommet de la Terre», soulève beaucoup d'interrogations, y compris d'ordre philosophique mais elle n'a droit qu'à un bref jugement de valeur négatif, y compris à propos des «zones humides [qui] sont sacralisées depuis la convention de Ramsar, bien qu'elles ne soient pas bonnes par essence» et dont "leur "assainissement" a été un grand progrès sanitaire», sans compter qu'elles sont en plus des «lieux de production de méthane». Prise de position assurément étrange à une époque où les zones humides sont reconnues comme hauts lieux de biodiversité procurant de multiples services et bienfaits aux sociétés humaines.

Mon troisième commentaire porte sur le contenu scientifique du livre sous le rapport de ces trois dimensions indissociables pour ce champ de recherche que sont l'écologie, l'évolution et les sciences de la conservation. La faible pénétration d'Alain Pavé dans la littérature, en particulier celle de «l'intelligence écoévolutive moderne» dont un magnifique corpus théorique s'est construit au cours des deux ou trois dernières décennies, entraîne le discours dans des considérations d'un autre âge, par exemple à propos des concepts d'écosystème, d'habitat, de niche, de communauté biotique ou encore de "stratégies d'histoires de vie» dont la puissance heuristique et la fécondité en termes d'écologie évolutive ont été amplement démontrées, dès les années 1990 dans les ouvrages fondateurs de Stephen Stearns (1992) et de Derek Roff (1992) pour ne citer qu'eux. À rebours de cette réalité, la communauté des scientifiques est accusée d'être fixiste, voire créationniste: «comment parler de diversité du vivant sans recourir à ce qui explique cette diversité, s'insurge l'auteur, sans introduire l'idée d'évolution, de changement permanent, c'est-à-dire, sans avoir une approche d'écologie évolutive ». Un simple survol de la littérature démontre à l'envi qu'une perspective évolutive imprègne aujourd'hui tous les travaux d'écologie, y compris les recherches en écologie fonctionnelle à propos de laquelle l'auteur écrit que «la biodiversité fonctionnelle a longtemps prévalu, au point qu'implicitement, et sans doute involontairement, c'est une vision quasi fixiste qui prédomine». Propos surprenants alors que tous les chercheurs considèrent aujourd'hui, et ce depuis longtemps, que l'expression "écologie évolutive» est pléonastique, tant le fait évolutif imprègne toutes les fonctions d'un organisme, à tous les stades de sa vie, y compris par des mécanismes longtemps insoupçonnés comme l'épigénétique dont on mesure aujourd'hui l'importance dans l'adaptation des organismes aux changements. "Nothing in biology makes sense except in the light of evolution 》 s'écriait Dobzhansky en 1973, propos paraphrasé par Begon, Harper et Townsend qui répliquaient en 1986 que «Very little in evolution makes sense except in the light of ecology» (Begon et al., 1986).
Le tout exprimé de manière conclusive par Endler (1992) pour qui «Ecology and evolution are so closely interrelated that they should not be regarded as separate fields ». Tous les chercheurs sont aujourd'hui convaincus de cette réalité et utilisent régulièrement des expressions telle que «dynamique ou théorie éco-évolutive».

Tout cela pour dire qu'accuser les chercheurs en écologie d'être fixistes est d'autant plus inattendu que les marqueurs moléculaires sont couramment mis à contribution pour étudier et démontrer le fait évolutif à tous les niveaux d'intégration biologique, des gènes aux paysages et à toutes les échelles de temps et d'espace. D'autant plus que les méthodes modernes de séquençage de l'ADN à haut débit, de génomique et de paléogénomique, ouvrent des perspectives encore inconnues il y a quelques années.

Se disant confiant dans la solidité des modèles et dans le «cadre théorique puissant» qu'ils offrent, Alain Pavé en arrive à soutenir que les «roulettes» biologiques qu'ils démontrent amènent à conclure «qu'au total les systèmes vivants tiennent plus d'un immense bazar que d'assemblages et de fonctionnements intangibles et réguliers », affirmation que ne manqueront pas de lire avec étonnement les spécialistes de l'écologie moderne des communautés qu'un solide corpus théorique a rénovée de fond en comble, en partie grâce à la mise à profit de la théorie des réseaux qui permet, entre autres, de connecter fonctionnellement et quantitativement les relations entre les compartiments végétaux et animaux de la biodiversité.

Sur la forme, le style délibérément provocateur et empreint de dérision traduit d'emblée la teneur d'un discours qui reste en permanence une critique radicale, allant jusqu'à la caricature, de la manière dont la communauté scientifique traite de ce sujet, stigmatisant même les publications scientifiques «qualifiées de sérieuses ». Le texte volontiers satirique fustige la délectation des médias pour les nouvelles catastrophistes, conteste l'effondrement des populations d'oiseaux et d'insectes, y compris dans les milieux agricoles intensifs, réfute les effets des dérèglements climatiques sur la biodiversité, bref se positionne à contre-courant de la doxa sur les grands déterminants de ce qu'on appelle le «changement global». Les écrits des acteurs de la biodiversité sont systématiquement contestés, y compris ceux de certains auteurs comme Paul et Anne Ehrlich qui sont «immensément faux» (sic!) et témoignent d'un malthusianisme affirmé, frisant l'eugénisme. En stigmatisant nommément, et de cette façon, le couple Ehrlich unanimement admiré et respecté de la communauté scientifique - une ligne rouge est franchie -, Alain Pavé englobe l'ensemble des chercheurs, y compris l'auteur de ces lignes, engagés dans des problématiques de conservation mais considérés comme des «donneurs 
de leçons». L'auteur conclut son livre par ce dernier message: "Oui, on peut faire de la biodiversité si l'on s'écarte des sentiers trop fréquentés, devenus banals et quelque peu ravinés des discours dominants actuels ».

\section{Une nécessaire pluralité d'approches}

L'événement que constitue la publication d'un tel livre est une occasion de revenir sur quelques fondements de la démarche scientifique telle qu'elle s'est progressivement construite dans le domaine de l'écologie évolutive appliquée à des problématiques de biodiversité et de sa conservation. Une confiance illimitée dans les modèles, ceux de l'auteur, aux dépens d'autres approches aussi puissantes que les approches moléculaires de nouvelle génération, sans parler de la puissance d'investigation que permet la miniaturisation des outils de recherche embarqués sur le vivant pour enregistrer en continu ses performances physiologiques ou comportementales, est en soi un phénomène intéressant. Car il soulève des questions d'ordre épistémologique sur la nature de la recherche scientifique et, dans le cas présent, du développement de l'écologie. Il est vrai que par une malencontreuse conjonction de cercles vicieux d'ordre culturel et sociologique, l'écologie a longtemps été privée, contrairement à d'autres sciences parfois considérées comme plus «nobles», de solides fondements théoriques (Rossberg et al., 2019). Mais ces temps sont révolus et l'écologie théorique dispose aujourd'hui d'un arsenal d'outils puissants qui permettent d'expliquer de manière cohérente et intégrée les structures écologiques qui s'emboîtent le long d'un continuum allant des populations locales aux assemblages d'espèces et aux écosystèmes.

De nombreux théoriciens sont venus à l'écologie «de l'extérieur ». Qu'ils soient chimistes, physiciens, mathématiciens, statisticiens, biométriciens, généticiens, chacun avec ses façons de penser, d'écrire, de nommer les choses et de communiquer, ils ont involontairement compliqué et obscurci la communication au point de ne pas voir la complémentarité des idées parce qu'elles paraissaient ne pas avoir de liens alors qu'elles sont constitutives de ce grand édifice complexe mais unitaire qu'est l'écologie théorique. Ce propos peut être illustré par l'histoire d'un champ thématique majeur en écologie, celui de la biologie des populations. Ce champ de recherche fut abordé dans les années 1950 à partir d'une cinétique démographique où les individus n'étaient que des unités ou "pions interchangeables», avec les célèbres controverses sur la densité dépendance versus la densité indépendance des populations qui ont méchamment opposé Lack (1954) et Andrewartha et Birch (1954) comme l'illustrent les pages savoureuses (290-299) de l'ouvrage de Lack (1966) Population studies of birds. Puis, la découverte, dans les années
1960, d'une grande variabilité entre individus dans leurs compétences en termes de fitness déboucha sur la célèbre, aujourd'hui dépassée, rhétorique de stratégies démographiques avec ses heures de gloire dans les années 1970 (Stearns, 1992 ; Roff, 1992). Aujourd'hui, la génétique puis la génomique des populations, la biométrie, la théorie des jeux, la théorie des métapopulations sont autant d'approches qui se sont longtemps ignorées, voire opposées, avant de participer toutes ensemble d'une construction cohérente de la biologie des populations. Dans un autre ordre d'idées, la non-linéarité et une bonne dose de stochasticité dans la réponse d'une variable aux facteurs qui la déterminent relèvent de mécanismes abordés de différentes manières selon qu'on fait appel à la théorie des jeux ou à différents types de modélisation mathématique formulés de manière assez générale pour s'accommoder des composantes idiosyncrasiques de tout système vivant. Ces approches relèvent de compétences et tournures d'esprit très différentes de celles qui caractérisent la recherche empirique mais les passerelles entre les deux sont pratiquement inexistantes. Chaque branche thématique ayant longtemps campé sur ses idées, souvent à l'exclusion des autres, toute synthèse combinant les différentes approches fut laborieuse, même lorsqu'il s'agit d'un même objet de recherche légitimement regardé avec des lunettes différentes.

\section{L'écologie, une science qui se cherche}

Parce qu'elle est multiforme et ambiguë en raison de nombreux amalgames, notamment avec l'écologisme, la question de savoir si l'écologie est vraiment une science est parfois posée. La «nébuleuse écologique» est un domaine de recherche vaste, difficile à circonscrire, au point que des dizaines de définitions lui ont été données. Pour beaucoup, certaines disciplines qui relèvent de l'écologie comme la biodiversité ou la biogéographie sont des sciences un peu bizarres, vagues, voire ésotériques qui restent en marge du panthéon des sciences nobles. Si bien que Malte Ebach (2003) se demande si le mot biogéographie n'est pas un "dirty word». Les disciplines dites «écologiques» doivent aussi la difficulté de leur identité aux divergences de vues qui sont longtemps restées tenaces entre écoles de pensée et de recherche. À la différence de sciences «nobles» comme la génétique formelle ou la physique, qui reposent respectivement sur la théorie évolutionniste néodarwinienne et sur celle de la relativité, l'écologie n'a pas vraiment réussi à élaborer un corpus théorique auquel aurait adhéré l'ensemble de la communauté scientifique. Si le concept d'écosystème définit bien une unité à laquelle tous les écologues se réfèrent, les méthodes ainsi que la terminologie utilisées pour l'étudier sont loin de faire l'unanimité. Le livre dont il est question ici, illustration d'une vision partielle donc partiale de la 
biodiversité, plaide par ses insuffisances mêmes, pour l'urgente nécessité d'un solide support théorique. Cette nécessité est régulièrement rappelée, encore récemment par Marquet et al. (2014), par Courchamp et al. (2015) et par Pásztor et al. (2016). Compendium d'un vaste spectre d'approches et de connaissances résultant d'une combinatoire d'observation et de modélisation, la théorie ne peut se construire que par la communication et le partage d'expériences complémentaires (Courchamp et Bradshaw, 2018). Pénétrer vraiment dans l'intimité d'une discipline aussi multiforme que l'écologie nécessite aussi de s'immerger dans la littérature. S'il est matériellement impossible de lire tout ce qui est écrit chaque année sur un sujet comme le nôtre, il existe de nombreux moyens pour accéder à l'essentiel de ce qu'il faut savoir. Partielle, partiale, et très franco-française, même si les prestigieux périodiques que sont Nature et Science sont convoqués à l'exclusion des autres, l'immersion de l'ouvrage dont il est question ici dans la littérature est très superficielle même si, s'appuyant sur une bibliographie de 233 titres, Alain Pavé affirme utiliser les connaissances les plus solides. Aucune référence n'est faite aux milliers d'articles publiés chaque année par des milliers d'auteurs dans la demi-douzaine au moins de grandes revues internationales sur les grands sujets d'intérêt qui concernent la biodiversité, ni dans les grandes revues qui font périodiquement le point sur l'état des connaissances en écologie et en évolution, donc en biodiversité, comme Trends in Ecology and Evolution. On ne peut que conseiller à l'auteur de consulter l'article de Frank Courchamp et Corey Bradshaw (2018) où il pourrait piocher de quoi apprendre le minimum de ce qu'il faut savoir pour traiter cet immense sujet.

\section{L'émergence de l'écologie scientifique}

Si l'écologie a eu du mal à s'imposer en France comme discipline respectable, c'est parce qu'elle a mis beaucoup de temps à s'affranchir de l'histoire naturelle dont elle est l'héritière, à se démarquer de l'écologisme militant et à s'intégrer résolument dans une démarche évolutive. On trouve tous ces «péchés de jeunesse» dans le livre dont il est question ici qui, faute d'avoir su pénétrer de plain-pied dans l'écologie moderne, témoigne de visions naïves et totalement dépassées, par exemple sur la « conception organiciste de la nature, bien visible dans le mythe de Gaïa», ou sur le fait que la biodiversité se réduit pour ceux qui l'étudient à une « comptabilité d'un nombre d'espèces », ce qui revient à tomber dans "l'effet "tonneau des Danaïdes" du dénombrement des espèces ", ou encore sur la notion de dette écologique alors que l'auteur n'a visiblement aucune idée du sens donné au mot « dette» en biologie de la conservation. Alain Pavé souligne qu'on compte ce qui disparaît mais pas ce qui apparait. Sauf que le solde du différentiel entre spéciation et extinction qu'on appelle en biogéographie historique le «taux de diversification » opère à des échelles de temps qui n'ont rien à voir avec celles dont parle l'auteur. Que la biodiversité ait augmenté au cours de l'histoire de la vie, personne n'en disconvient, mais le tempo et le mode de cette augmentation opèrent à des échelles de temps bien différentes de celles auxquelles ont lieu les sautes de biodiversité occasionnées par chacun des grands spasmes d'extinction qui se sont succédé entre l'Édiacarien (635-541 millions d'années) et la fin du Crétacé (65 millions d'années). Or ce sont ces crises qui ont «sculpté» la biodiversité par l'enchaînement des assemblages d'espèces et l'apparition de nouveaux «plans de vie» offerts à l'évolution par un large éventail de nouveaux possibles. Mais ce qui nous préoccupe aujourd'hui de manière tangible, c'est bien le spasme actuel qui laisse l'auteur dubitatif alors que ce que nous perdons aujourd'hui - on parle de près de la moitié des espèces d'ici le mitan de ce siècle - sera perdu "pour toujours » puisqu'on sait qu'il faut des millions d'années pour que la biodiversité se reconstitue après une grande crise et qu'il n'y a pas de raison pour que l'espèce humaine vive plus longtemps que les autres dont la durée de vie est estimée, à partir des registres fossiles, entre un et dix millions d'années. Certains raisonnements fantaisistes et totalement irréalistes montrent bien que l'ouvrage ne rend pas justice aux recherches récentes de biogéographie historique qui plongent dans le passé profond grâce aux phylogénies moléculaires. Telle cette remarque que "[sur] la base "optimiste" de 100000 ans [pour le processus de spéciation], soit $1 / 40000$ de $4.10^{9}$ [1'histoire de la vie sur la Terre], on en déduit qu'il y aurait 40000 espèces sur la planète en supposant que depuis l'émergence de la vie aucune n'ait disparu. Or, on parle de dizaines de millions d'espèces actuellement. On surestime drastiquement les extinctions et l'on sousestime, non moins drastiquement, les apparitions, tout en restant souvent fantaisistes sur les estimations globales. »... ! Là encore, on ne peut que suggérer quelques références éclairantes sur de tels sujets, par exemple les travaux de Michael Benton (1995, 1997).

\section{Biodiversité et conservation}

Quant à l'immense domaine de la conservation auquel renvoie le concept de biodiversité, en écrivant que « conserver, c'est fixer arbitrairement et artificiellement et ce n'est pas forcément le meilleur moyen de maintenir la biodiversité, et en tout cas pas de l'augmenter», ou le «courant dominant [est] essentiellement conservationniste, de nature plutôt fixiste », l'auteur de ce livre ignore ce que sont les sciences de la conservation dont il ne cite aucun des textes fondateurs. Il faut dire que, sans jamais vraiment l'écrire, Alain Pavé ne croit pas à l'érosion de la 
biodiversité dont le constat et la dénonciation seraient un mythe « pour prendre une parabole biblique très habituelle chez les prêcheurs de l'apocalypse biosphérique». À l'évidence, il rejoint une toute petite communauté de chercheurs qui ne croient pas à l'érosion de la biodiversité, du moins à certaines de ses composantes, reliant ainsi, sans les citer, d'autres «écolosceptiques» comme Bjorn Lomborg (2001), auteur de The skeptical environmentalist. Measuring the real state of the world.

\section{Reconstruire l'écologie?}

Oui, bien sûr, car si la biodiversité n'est qu' « un motvalise, une auberge espagnole, un attracteur pour le moins étrange, un concept évanescent, quelquefois gluant, etc.», accessoirement une «biodiversité-alibi» utilisée pour contrecarrer des projets d'aménagement, il est effectivement urgent de reconstruire l'écologie. " $\mathrm{Au}$ stade actuel, nous sommes devant un dilemme, à la fois convaincu de la portée de ce que représente le terme "biodiversité" et réservé face à l'usage qui en est fait». Fort de son analyse, Alain Pavé propose une «reconstruction » de l'écologie, mais il se limite à cette phrase (p. 276) «l'écologie, en tant que discipline scientifique, ne peut pas se contenter de compter et de collectionner, elle doit changer en amplifiant les dimensions fonctionnelles et en développant encore plus les aspects évolutifs, très positifs pour l'étude de la biodiversité elle-même et de sa dynamique $» .$. . Ce qui est écrit dans ces lignes montre que, fort heureusement, la communauté scientifique n'a pas attendu ce livre pour le faire.

Finalement, quel dialogue pourrait-on imaginer entre l'auteur de cet ouvrage et les experts de l'IPBES (Plateforme intergouvernementale scientifique et politique sur la biodiversité et les services écosystémiques) relevant de 132 pays qui, réunis à Paris, ont lancé le 6 mai 2019 , donc quelques semaines après la publication de ce livre, une "alerte rouge» confirmant ce qu'ont publié plus de 15000 scientifiques dans la revue BioScience en 2017, à savoir que «la destruction de l'environnement pousse les écosystèmes au-delà de leurs capacités à entretenir le tissu de la vie»? (Ripple et al., 2017). Décidément, les « idées fausses » dénoncées dans ce livre sont des idées vraies pour l'immense majorité des chercheurs impliqués dans ce champ de recherche! Ainsi va la recherche: dans un ouvrage récent consacré à la «vie secrète de la science», Jeremy Baumberg (2018) s'interroge sur la manière dont fonctionne le « cirque des publications scientifiques » où chaque auteur est immergé dans cet écosystème si particulier qu'est la communauté des scientifiques, avec ses règles, ses fables, ses marottes et ses fantasmes. Au lecteur de choisir où ce livre se situe dans ce grand cirque.

\section{Références}

Andrewartha H.G., Birch L.C., 1954. The distribution and abundance of animals, Chicago, University of Chicago Press.

Baumberg J.J., 2018. The secret life of science. How it really works and why it matters, Princeton, Princeton University Press.

Begon M., Harper J.L., Townsend C.R., 1986. Ecology: Individuals, populations and communities, Sunderland, Sinauer.

Benton M.J., 1995. Diversification and extinction in the history of life, Science, 268, 52-58.

Benton M.J., 1997. Models of the diversification of life, Trends in Ecology \& Evolution, 12, 12, 490-495, https://doi.org/ 10.1016/S0169-5347(97)84410-2.

Blondel J., 2012. L'archipel de la vie. Essai sur la diversité biologique et une éthique de sa pratique, Paris, BuchetChastel.

Courchamp F., Bradshaw C.J.A., 2018. 100 articles every ecologist should read, Nature Ecology \& Evolution, 2, 395401, https://doi.org/10.1038/s41559-017-0370-9.

Courchamp F., Dunne J.A., Le Maho Y., May R.M., Thébaud C., Hochberg M., 2015. Fundamental ecology is fundamental, Trends in Ecology \& Evolution, 30, 1, 9-16, https://doi.org/10.1016/j.tree.2014.11.005.

Dobzhansky T., 1973. Nothing in biology makes sense except in the light of evolution, The American Biology Teacher, 35, 3, 125-129.

Ebach M.C., 2003. Biogeography, a dirty word?, Biologist, 50, $1,48$.

Endler J., 1992. Signals, signal conditions, and the direction of evolution, The American Naturalist, 139, S125-S153.

Lack D., 1954. The natural regulation of animal numbers, Oxford, Clarendon Press.

Lack D., 1966. Population studies of birds, Oxford, Oxford University Press.

Lomborg B., 2001. The skeptical environmentalist. Measuring the real state of the world, Cambridge, Cambridge University Press. Trad. fr. : L'écologiste sceptique. Le véritable état de la planète, Paris, Le Cherche midi, 2004.

Marquet P.A., Allen A.P., Brown J.H., Dunne J.A., Enquist B. J., Gillooly J.F., Gotawy P.A., Green J.L., Harte J., Hubbell S.P., O’Dwyer J., Okie J.G., Ostling A., Ritchie M., Storch D., West G.B., 2014. On theory in ecology, BioScience, 64, 8, 701-710, https://doi.org/10.1093/biosci/biu098.

MEA (Millennium Ecosystem Assessment), 2005. Ecosystems and human well-being. Biodiversity synthesis. Washington, World Resource Institute.

Pásztor L., Botta-Dukát Z., Magyar G., Czárán T., Meszéna G., 2016. Theory-based ecology. A Darwinian approach, Oxford, Oxford University Press.

Ripple W.J., Wolf C., Newsome T.M., Galetti M., Alamgir M., Crist E., Mahmoud M.I., Laurance W.F. et 15364 scientifiques signataires venant de 184 pays, 2017. World scientists warning to humanity: a second notice, Bioscience, 67, 12, 1026-1028. 
Roff D.A., 1992. The evolution of life histories, New York, Chapman \& Hall.

Rossberg A.G., Barábas G., Possingham H.P., Pascual M., MarquetP. A., Hui C., Evans M.R., Meszéna G., 2019. Let’s train more theoretical ecologists here is why, Trends in Ecology \& Evolution, 34, 9, 759-762, https://doi.org/10.1016/j.tree.2019.06.004.

Stearns S.C., 1992. The evolution of life histories, Oxford, Oxford University Press.

Citation de l'article : Blondel J. À propos de l'ouvrage d'Alain Pavé Comprendre la biodiversité. Vrais problèmes et idées fausses. Nat. Sci. Soc. 28, 1, 73-80. 